\title{
SCREENING OF PLANTS FOUND IN AMAZONAS STATE FOR LETHALITY TOWARDS BRINE SHRIMP
}

\author{
Etienne Louis Jacques QUIGNARD, Adrian Martin POHLIT, Sergio Massayoshi \\ NUNOMURA, Ana Cristina da Silva PINTO, Elba Vieira Mustafa dos SANTOS, Sabrina \\ Kelly Reis de MORAIS, Alexandre Mascarenhas ALECRIM, Andreza Cristiana da Silva \\ PEDROSO, Barbara Rachel Brito CYRINO, Christiane Santana de MELO, Ellen Kathryn \\ FINNEY, Erika de Oliveira GOMES, Katiuscia dos Santos de SOUZA, Laura Cristina Pereira \\ de OLIVEIRA, Luciana de Castro DON, Luiz Francisco Rocha e SILVA, Maria Mireide \\ Andrade QUEIROZ, Marycleuma Campos HENRIQUE, Mirian dos SANTOS, Patrícia de \\ Souza PINTO, Suniá Gomes SILVA*
}

ABSTRACT: 226 methanol and water extracts representing 74 mainly native plant species found in Amazonas State, Brazil, were tested at a standard concentration of $500 \mu \mathrm{g} / \mathrm{mL}$ for lethality towards larvae of the brine shrimp species Artemia franciscana. Several cytotoxic plant species were identified in this work: Aspidosperma marcgravianum, A. nitidum, Croton cajucara, Citrus limetta, Geissospermum argenteum, Minquartia guianensis, Piper aduncum, P. amapense, P. capitarianum, P. tuberculatum and Protium aracouchini. The results were analyzed within the context of the available traditional knowledge and uses for these plants.

KEYWORDS: Artemia franciscana, cytotoxicity, amazonian plants.

\section{SCREENINGDE PLANTAS ENCONTRADAS NO ESTADO DO AMAZONAS PARA LETALIDADE AO MICROCRUSTÁCEO Artemia franciscana}

RESUMO: Foram testados 226 extratos metanólicos e aquosos de 74 espécies vegetais (a maioria nativas) encontradas no Estado do Amazonas, Brasil, para letalidade às larvas da espécie microcrustácea Artemia franciscana na concentração teste de $500 \mu \mathrm{g} / \mathrm{mL}$. Várias espécies citotóxicas foram identificadas nesse estudo: Aspidosperma marcgravianum, $A$. nitidum, Citrus limetta, Croton cajucara, Geissospermum argenteum, Minquartia guianensis, Piper aduncum, P. amapense, P. capitarianum, P. tuberculatum e Protium aracouchini. Os resultados foram analisados levando em consideração as informações populares, quando dis poníveis.

PALAVRAS-CHAVE: Artemia franciscana, citotoxicidade, plantas amazônicas.

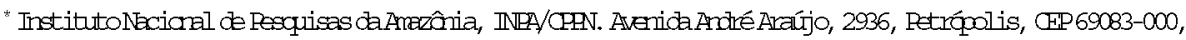
Maras, AVL. Brasil, enail: apphlitainpa.grv.br.
} 


\section{INTRODUCTION}

Amazonian plant species are potential sources of phytotherapeutic agents, nutraceuticals, and leads for the development of new drugs (duke, 1994). Experience has shown that an obvious starting point for research in these areas is the literature on traditional knowledge of plants of the Amazon region (grenand, 1987; lorenzi, 1998; pio Corrêa, 1978; revilla, 2002; schultes, 1990; silva, 1977). These texts identify plants described by their users as having medicinal and other useful properties. However, for many plants there is no relevant literature available and so biological activity must be found using more direct methods such as farmacological testing or screening.

The brine shrimp lethality assay consists of exposing larvae to plant extract in saline solution and lethality is evaluated after a day. The commercial availability of inexpensive brine shrimp eggs, the low cost, safety and ease of performing the assay, as well as the lack of a need for special equipment make this a very helpful bench-top tool for the phytochemistry laboratory (mcLaughlin, 1991). First developed by meyer (1982), this assay has wide application in research towards the discovery of cytotoxic and other active principles present in plant extracts (mongelli, 1996). For example, a very positive correlation between the lethality to brine shrimp and antitumoral activity has been established by researchers working on the development of new anti-cancer drugs from plants at the National Cancer Institute (NCI) in the United States (anderson, 1991). This correlation is considered so good that lethality to brine shrimp is recommended by these authors as an effective pre-screen to existing cytotoxicity and antitumor assays. More recently, it has been shown that there is a very good correlation between the median lethal concentrations $\left(\mathrm{LC}_{50}\right)$ of plant extracts to brine shrimp larvae and the median lethal doses $\left(\mathrm{LD}_{50}\right)$ of these same extracts, administered orally in mice (Parra, 2001). A number of other studies have demonstrated the use of the brine shrimp assay to screen plants popularly used as pesticides (Fatope, 1993), plants having ethnomedical uses related to cancer (mongelli, 1996), and tropical plants used medicinally (together with screening for larvicidal, fungicidal, and molluscicidal activity in cepleanu (1994)). Lastly, this assay has been used successfully to biomonitor the isolation of cytotoxic (siqueira, 2001), antineoplastic (Badaway, 1997), antimalarial (pérez, 1997), insecticidal (oberlies, 1998) and anti-feedant (labbe, 1993) compounds from plant extracts.

In what follows, the results of a screening of methanol and water extracts of some common medicinal and other plant species (collected in Amazonas State, Brazil) for lethality towards Artemia franciscana larvae are presented.

\section{METERIALS AND METHODS}

Collection and drying of plants. Plants were collected in or near Benjamin Constant (located near the Brazilian border with Peru and Colombia), Novo Airão (110 km from Manaus) and Manaus (at the INPA Adolfo Ducke Reserve). Identification was performed by employees at the Herbarium at the University of Amazonas (Manaus, Amazonas) or at the Herbarium at the National Institute for Amazon Research (Instituto Nacional de Pesquisas da Amazônia, INPA). Plants were air dried in the shade and later separated by part (leaf, stem, bark, etc.).

Preparation of water extracts. Extraction was performed by infusion of the ground plant material in boiling water for 15 minutes after which the mixture was filtered and the filtrate evaporated to dryness under vacuum and then freezedried. The extracts were stored in a freeezer $\left(-19^{\circ} \mathrm{C}\right)$.

Preparation of methanol extracts. A known mass of each ground plant underwent con- 
tinuous liquid-solid extraction with methanol in a Soxhlet apparatus for three consecutive $6 \mathrm{~h}$ periods (total $18 \mathrm{~h}$ ). After each $6 \mathrm{~h}$ interval, the extract was removed and the Soxhlet apparatus was recharged with fresh methanol to avoid undue exposure of the extracts to heat during extraction. The combined methanol extracts were evaporated and stored as described for the water extracts.

Preparation of stock saline solution. $\mathrm{Ni}$ trate, phosphate and silicate free saline stock solution was prepared by dissolving mineral enriched sea salt (SERA, Germany) in de-ionized water at a concentration of $35 \mathrm{~g} / \mathrm{L}$.

Hatching of brine shrimp larvae. Brine shrimp eggs (Brine Shrimp Direct, U.S.A.) were sprinkled into a Petri dish containing saline solution and left for two days at $26 \pm 1{ }^{\circ} \mathrm{C}$ under an incandescent lamp. After this period, the eggs had hatched and second instar nauplii (larvae) were observed to be swimming near the light source.

Sample preparation. Methanol extracts were reconstituted in DMSO and water extracts in deionized water at a standard concentration of $50 \mathrm{mg} / \mathrm{mL}$ using sonication, shaking and/or mild heating.

Brine shrimp lethality assay. The wells of 24-well microtiter plates were pre-filled with $1.8 \mathrm{~mL}$ of saline solution. Next, 10 two-day old nauplii (second instar larvae) in a minimum of saline solution, $20 \mu \mathrm{L}$ of each reconstituted extract solution and stock saline solution were added to give a final volume of $2.0 \mathrm{~mL}$ in each well. This corresponds to a $500 \mu / L$ extract concentration in each experimental well. Each extract was tested in triplicate. Control wells received $20 \mu \mathrm{L}$ of the corresponding solvent (DMSO or de-ionized water) used for the tested samples, instead of extract, and had the same final volume $(2.0 \mathrm{~mL})$ as experimental wells.
The plates were allowed to stand in the $a b$ sence of direct light (to avoid possible false positive results due to the generation of phototoxic compounds) at room temperature and mortality was evaluated after $24 \mathrm{~h}$ as the percentage of dead larvae present after $24 \mathrm{~h}$ relative to live larvae present originally.

\section{RESULTS AND DISCUSSION}

Information on the native and exotic species studied is presented in Table 1. Many of these plants were collected as part of an ongoing research program on the biological activity and chemistry of Amazonian antimalarial plants used in traditional medicine and include the barks of carapanaúba, acariquara branca, taperebá (also frequently called cajá), abiu, castanha do Pará (Brazil nut) trees and other plant species. Other species, such as $A s p i$ dosperma spp., Piper spp. and Cassia spp., were collected following a chemosystematic approach, i.e. they belong to genera from which cytotoxic, antimalarial and other interesting medicinal substances have been isolated. When possible, several parts of the plant were col lected, not restricting the parts collected to those used traditionally. This allows for a more complete analysis and understanding of the cytotoxicity of the plants and the part(s) of the plants where cytotoxic substances are concen trated.

Table 2 shows the results of the screening of methanol and water extracts of plants using the brine shrimp assay. In all, $226 \mathrm{ex}$ tracts representing 74 species were screened for lethality to brine shrimp nauplii and 24.3 $\%$ (55 extracts) had lethalities > 20\%, $14.6 \%$ (33 extracts) had lethalities $>50 \%$, while only $8.8 \%$ (20 extracts) were considered highly ac tive, having lethalities $>90 \%$ to brine shrimp. 
Table 1. Ethnobotanic information for the plants studied.

\begin{tabular}{|c|c|c|c|c|}
\hline $\begin{array}{l}\text { FAMILY } \\
\text { Scientific name }\end{array}$ & $\begin{array}{l}\text { Common } \\
\text { name }\end{array}$ & $\begin{array}{l}\text { Part(s) } \\
\text { used }\end{array}$ & $\begin{array}{l}\text { Medicinal and other use(s) / } \\
\text { property(-ies) }\end{array}$ & Ref \\
\hline \multicolumn{5}{|l|}{ ANACARDIACEAE } \\
\hline Spondias mombin $\mathrm{L}$. & taperebá, cajá & $\begin{array}{l}\text { leaf, fruit, } \\
\text { root, bark }\end{array}$ & $\begin{array}{l}\text { treatment of malaria, fever, } \\
\text { diarrhea; vaginal infections }\end{array}$ & 1,2 \\
\hline \multicolumn{5}{|l|}{ ANNONACEAE } \\
\hline $\begin{array}{l}\text { Bocageopsis multiflora (Mart.) R. E. Fries } \\
\text { APIACEAE }\end{array}$ & $\begin{array}{l}\text { envira } \\
\text { surucucu }\end{array}$ & wood & $\begin{array}{l}\text { construction, tools, } \\
\text { ornament, reforestation }\end{array}$ & 3,4 \\
\hline Eryngium foetidum $\mathrm{L}$. & chicória & $\begin{array}{l}\text { leaf, root, } \\
\text { stem, fruit }\end{array}$ & $\begin{array}{l}\text { perfumary, treatment of } \\
\text { diarrhea, fever, headaches }\end{array}$ & 2,5 \\
\hline \multicolumn{5}{|l|}{ APOCYNACEAE } \\
\hline Aspidosperma araracanga Marcondes-Ferreira & & & & 4 \\
\hline Aspidosperma desmanthum Benth. ex Müll. Arg. & araracanga & leaf & febrifuge & 4,6 \\
\hline Aspidosperma marcgravianum Woodson & carapanaúba & $\begin{array}{l}\text { wood, } \\
\text { bark }\end{array}$ & $\begin{array}{l}\text { construction; treatment } \\
\text { malaria diabetes, cancer }\end{array}$ & 1,4 \\
\hline Aspidosperma nitidum Benth. ex Müll. Arg. & carapanaúba & $\begin{array}{l}\text { wood, } \\
\text { bark, latex }\end{array}$ & $\begin{array}{l}\text { construction; treatment } \\
\text { leprosy, malaria, cancer }\end{array}$ & 1,4 \\
\hline Aspidosperma sandwithianum Markgr. & & & & 4 \\
\hline Aspidosperma schultesiiWoodson & & bark & $\begin{array}{l}\text { termite protector; } \\
\text { antimycotic; febrifuge }\end{array}$ & 4,7 \\
\hline Aspidosperma spruceanum Benth. ex Müll. Arg. & & wood & construction & 3,4 \\
\hline Aspidosperma vargasii A. DC. & & & $\begin{array}{l}\text { fever; wound treatment, } \\
\text { insect bites }\end{array}$ & 2,7 \\
\hline Aspidosperma sp. & & & & 4 \\
\hline Geissospermum argenteum Woodson & $\begin{array}{l}\text { acariquara } \\
\text { branca }\end{array}$ & bark & fever; malaria & 2,4 \\
\hline Himatanthus sucuuba (Spruce ex Müll. Arg.) Woodson & sucuuba & latex & $\begin{array}{l}\text { fever, bone fractures, } \\
\text { tooth aches }\end{array}$ & 1,4 \\
\hline \multicolumn{5}{|l|}{ ARECACEAE } \\
\hline Euterpe oleracea Mart. & açaizeiro & $\begin{array}{l}\text { root, fruit, } \\
\text { seed }\end{array}$ & $\begin{array}{l}\text { beverage; treatment jaundice; } \\
\text { fever }\end{array}$ & 1 \\
\hline Mauritia flexuosa L.f. & buriti & fruit & food, beverage & 1,4 \\
\hline \multicolumn{5}{|l|}{ ASTERACEAE } \\
\hline Bidens bipinnatus $\mathrm{L}$. & $\begin{array}{l}\text { carrapicho } \\
\text { de agulha }\end{array}$ & & & 8 \\
\hline Spilanthes acmella (L.) Murray & jambú & flower & lung diseases, tuberculosis & 1 \\
\hline \multicolumn{5}{|l|}{ BIGNONIACEAE } \\
\hline Arrabidaea chica (Humb. \& Bonpl.) B. Verl. & crajirú & leaf & $\begin{array}{l}\text { dye, astringent; treatment } \\
\text { infections, inflammation }\end{array}$ & 1,5 \\
\hline Tabebuia incana A.H. Gentry & pau d'arco & bark & candidiasis; tumors & 1 \\
\hline Tabebuia serratifolia(Vahl) G. Nichol. & pau d'arco & $\begin{array}{l}\text { wood, } \\
\text { bark }\end{array}$ & $\begin{array}{l}\text { candidiasis; tumors; } \\
\text { fever; leishmaniosis }\end{array}$ & 1 \\
\hline \multicolumn{5}{|l|}{ BURSERACEAE } \\
\hline Protium aracouchini (Aubl.) Marchand & breuvermelho & resin & laxative & 9 \\
\hline \multicolumn{5}{|l|}{ DICHAPETALACEAE } \\
\hline Tapura amazonica Poepp. & tapura & leaf & toxic & 7 \\
\hline \multicolumn{5}{|l|}{ EUPHORBIACEAE } \\
\hline Croton cajucara benth. & sacaca & bark & $\begin{array}{l}\text { treatment of diabetes; diarrhea; liver } \\
\text { inflammation }\end{array}$ & 1 \\
\hline Croton lanjouwensis jabl. & dima & bark & fever & 2,10 \\
\hline Mabea subsessilis Pax \& K. Hoffim. & taquari & wood & & 4 \\
\hline Micrandra siphonioides Benth. & seringarana & wood & & 4 \\
\hline
\end{tabular}


Table 1. Ethnobotanic information for the plants studied (Continued).

\begin{tabular}{|c|c|c|c|c|}
\hline $\begin{array}{l}\text { FAMILY } \\
\text { Scientific name }\end{array}$ & $\begin{array}{l}\text { Common } \\
\text { name }\end{array}$ & $\begin{array}{l}\text { Part(s) } \\
\text { used }\end{array}$ & $\begin{array}{l}\text { Medicinal and other use(s)/ } \\
\text { property(-ies) }\end{array}$ & Ref \\
\hline \multicolumn{5}{|l|}{ EUPHORBIACEAE } \\
\hline Micrandropsis scleroxylon W.A. Rodrigues & piãozinho & wood & & \\
\hline Phyllanthus niruriL. & quebra-pedra & $\begin{array}{l}\text { whole } \\
\text { plant }\end{array}$ & $\begin{array}{l}\text { treatment of malaria, kidney } \\
\text { stones, liver disease }\end{array}$ & 1 \\
\hline Piranhea trifoliata Baill. & piranheira & wood & construction, carpentry & 11 \\
\hline \multicolumn{5}{|l|}{ GENTIANACEAE } \\
\hline Tachia grandiflora Maguire \& Weaver & & & & 4 \\
\hline \multicolumn{5}{|l|}{ LAMIACEAE } \\
\hline Ocimum micranthum Willd. & alfavaca & leaf & malaria & 2,5 \\
\hline \multicolumn{5}{|l|}{ LAURACEAE } \\
\hline Aniba canelilla (Kunth.) Mez. & preciosa & $\begin{array}{l}\text { bark, } \\
\text { seed, leaf }\end{array}$ & $\begin{array}{l}\text { perfumary, tonic; treatment } \\
\text { malaria, syphilis, dysentery }\end{array}$ & 5 \\
\hline \multicolumn{5}{|l|}{ LECYTHIDACEAE } \\
\hline Bertholletia excelsa Bonpl. & $\begin{array}{l}\text { castanha do } \\
\text { Pará }\end{array}$ & seed, bark & $\begin{array}{l}\text { food; cosmetics; treatmento } \\
\text { liver ailments, anemia }\end{array}$ & 1 \\
\hline Corythophora alta R. Kunth. & $\begin{array}{l}\text { ripeiro } \\
\text { (vermelho) }\end{array}$ & wood & & 4 \\
\hline Eschweilera bracteosa (Poepp. ex O. Berg) Miers & $\begin{array}{l}\text { matamatá } \\
\text { amarelo }\end{array}$ & mood & & 4 \\
\hline Gustavia elliptica S. A. Mori & mucurão & wood & construction, ornament & 4 \\
\hline \multicolumn{5}{|l|}{ LEGUMINOSAE: MIMOSOIDEAE } \\
\hline $\begin{array}{l}\text { Abarema floribunda (Spruce ex Benth.) Barneby \& } \\
\text { J.W. Grimes }\end{array}$ & & & & 4 \\
\hline \multicolumn{5}{|l|}{ LEGUMINOSAE: CAESALPINIOIDEAE } \\
\hline Cassia fastuosa Willd. ex Vogel & chuva-de-ouro & & ornament & \\
\hline Cassia siamea Lam. & acácia de Siam & & & \\
\hline Cassia spruceana Benth. & $\begin{array}{l}\text { mari-mari-da- } \\
\text { terra-firme }\end{array}$ & root & fever & 2,4 \\
\hline Senna occidentalis $(\mathrm{L}$.$) Link$ & mata-pasto & root, seed & $\begin{array}{l}\text { treatment fever, malaria, } \\
\text { asthma; abortive, toxic }\end{array}$ & 1,2 \\
\hline Senna reticulata (Willd.) H.S. Irwin \& Barneby & mata-pasto & leaf, flower & $\begin{array}{l}\text { liver diseases; stomach } \\
\text { aches; kidney inflammation }\end{array}$ & 1,2 \\
\hline \multicolumn{5}{|l|}{ LEGUMINOSAE: PAPILIONOIDEAE } \\
\hline Bowdichia nitida Spruce ex Benth. & sucupira & bark & $\begin{array}{l}\text { treatment of syphilis, } \\
\text { skin diseases }\end{array}$ & 7,4 \\
\hline Swartizia pronacencis Amsh. & mututi & & & 8 \\
\hline \multicolumn{5}{|l|}{ MENISPERMACEAE } \\
\hline Abuta grandifolia (Mart.) Sandwith. & cipó-de-bota & root, stem, leaf & $\begin{array}{l}\text { treatment of sterility, } \\
\text { malaria, anemia }\end{array}$ & 1 \\
\hline \multicolumn{5}{|l|}{ OLACACEAE } \\
\hline Minquartia guianensis Aubl. & $\begin{array}{l}\text { acariquara } \\
\text { vermelha }\end{array}$ & wood, bark & posts, civil construction; fish poison & 1 \\
\hline \multicolumn{5}{|l|}{ PHYTOLACCACEAE } \\
\hline Petiveria alliacea $\mathrm{L}$. & mucuracá & whole plant & $\begin{array}{l}\text { abortive; antispasmodic; antirheumatic; } \\
\text { antipyretic; diuretic }\end{array}$ & 1 \\
\hline Agonandra brasiliensis Miers ex Benth. \& Hook. f. & pau marfim & wood & tools, carpentry & 10 \\
\hline \multicolumn{5}{|l|}{ PIPERACEAE } \\
\hline Piper aduncum $\mathrm{L}$. & pimenta longa & leaf, fruit & $\begin{array}{l}\text { aromatic, styptic, antimicrobial, antimy- } \\
\text { cotic }\end{array}$ & 7 \\
\hline Piper amapense Yunck. & tapi-ipele & leaf, stem & treatment of dizziness & 9 \\
\hline
\end{tabular}


Table 1. Ethnobotanic information for the plants studied (Continued).

\begin{tabular}{|c|c|c|c|c|}
\hline $\begin{array}{l}\text { FAMILY } \\
\text { Scientific name }\end{array}$ & $\begin{array}{l}\text { Common } \\
\text { name }\end{array}$ & $\begin{array}{l}\text { Part(s) } \\
\text { used }\end{array}$ & $\begin{array}{l}\text { Medicinal and other use(s) / } \\
\text { property(-ies) }\end{array}$ & Ref \\
\hline \multicolumn{5}{|l|}{ PIPERACEAE } \\
\hline Piper cyrtopodum C. DC. & jacamim & leaf & aromatic baths & 10 \\
\hline \multicolumn{5}{|l|}{ Piper dilatatum Rich. } \\
\hline \multicolumn{5}{|l|}{ Piper erectipilum Yunck. } \\
\hline Piper hostmannianum (Miq.) C. DC. & cordoncillo & leaf & treatment of warts & 7 \\
\hline Piper tuberculatum Jacq. & pimenta longa & leaf & fish poison & 7,6 \\
\hline Pothomorphe peltata (L.) Miq. & caapeba & leaf & $\begin{array}{l}\text { diuretic, antipyretic and emetic; sudor- } \\
\text { ific }\end{array}$ & 1 \\
\hline \multicolumn{5}{|l|}{ POACEAE } \\
\hline Cymbopogon citratus (DC.) Stapf. & capim-santo & leaf, root & beverage; digestive; antipyretic & 1 \\
\hline \multicolumn{5}{|l|}{ RHAMNACEAE } \\
\hline Ampelozizyphus amazonicus Ducke & saracura-mirá & root, bark, leaf & $\begin{array}{l}\text { treatment of insect bites; depurative; } \\
\text { antimalarial }\end{array}$ & 1,2 \\
\hline \multicolumn{5}{|l|}{ RHIZOPHORACEAE } \\
\hline Cassipourea guianensis Aubl. & araçá bravo & & & 4 \\
\hline \multicolumn{5}{|l|}{ RUBIACEAE } \\
\hline Ladenbergia undata Klotzsch & quina-quina & & & \\
\hline Palicourea carymbifera (Müll. Arg.) Standl. & & bark & $\begin{array}{l}\text { treatment of persistent cough, chest } \\
\text { ailments }\end{array}$ & 7,4 \\
\hline Palicourea guianensis Aubl. & & root, leaf & styptic and hemostatic; vermifuge & 7 \\
\hline Palicourea virens (Poepp. \& Endl.) Standl. & & & & 4 \\
\hline \multicolumn{5}{|l|}{ RUTACEAE } \\
\hline Citrus limetta Risso & limeira de umbigo & & & 8 \\
\hline \multicolumn{5}{|l|}{ SAPOTACEAE } \\
\hline Micropholis venulosa (Mart. \& Eichler) Pierre & abiurana branca & fruit & food & 1 \\
\hline Pouteria caimito (Ruiz \& Pav.) Radlk. & abieiro & fruit, wood & food, construction & 3 \\
\hline Pouteriaguianensis Aubl. & abiurana, abieiro & fruit & food & 1 \\
\hline \multicolumn{5}{|l|}{ SIMAROUBACEAE } \\
\hline Picramnia Spruceana Engl. & sam panga & fruit & dye; treatment of skin irritation & 1 \\
\hline Simaba cedron Planch. & & & pungent & 4 \\
\hline \multicolumn{5}{|l|}{ SOLANACEAE } \\
\hline Physalis angulata $L$. & camapú & leaf, fruit, root & narcotic; diuretic; anti-inflamatory; & 1 \\
\hline \multicolumn{5}{|l|}{ VIOLACEAE } \\
\hline Rinorea guianensis Aubl. & falsa cupiuba & wood & construction & 4 \\
\hline Rinorea racemosa (Mart.) Kuntze & branquinha & wood & construction & 1 \\
\hline
\end{tabular}


The most active species were Aspidosperma marcgravianum, A. nitidum, Citrus limetta, Croton cajucara, Geissospermum argenteum, Minquartia guianensis, Piper tuberculatum, $P$. aduncum, $P$. amapense, $P$. capitarianum and Protium aracouchini. It is interesting that species such as Piper tuberculatum and Minquartia guianensis, which are traditionally used for their toxic properties, and Tapura amazonica, which is reportedly toxic, show significant lethality to brine shrimp. This result is similar in kind to that of Parra (2001) who found a correlation between the toxicity of orally administered plant extracts in mice and the lethal concentration of the extracts to brine shrimp.

Our systematic approach to screening of the genus Aspidosperma revealed that only the barks of a few species were active in the brine shrimp assay. This would seem to indicate that cytotoxic compounds are not generally distributed in the barks of the Aspidosperma species studied. The two species $A$. nitidum and A. marcgravianum, both popularly called carapanaúba, are traditionally used for the treatment of a number of diseases, including cancer. Since it is known that Aspidosperma species produce antitumor alkaloids such as ellipticine (Ohashi, 1996), the activity of these two species in the brine shrimp assay make them interesting as potencial sources of anticancer agents. Our results show that several Piper species are also active in the brine shrimp assay which is consistent with existing phytochemical knowledge of this genus as a source of cytotoxic and antitumor compounds (Parmar, 1997).

\section{CONCLUSION}

A number of cytotoxic plants were iden tified during this work. Several of them are now under biomonitored phytochemical analysis and should yield isolated cytotoxic components in due time. These results represent an important first step in a planned long term collaboration between labs at CPPN and those at the College of Medicine at the University of Ceará (Fortaleza, Ceará State, Brazil) for the discovery of anticancer compounds present in Amazonian plant species.

\section{ACKNOWLEDGEMENTS}

The authors proudly recognize the financial support provided by the Brazilian $\mathrm{Na}$ tional Council for Scientific and Technological Development (CNPq), grant nos. 520.354/990 and 550.260/01-3. The following people are thankful to these institutions for scholarships: ELJQ (DTI-MCT), SMN (DCR-CNPq), ACSP and EVMS (Mestr-CAPES), SKRM (AP-CNPq), BRBC and MCH (IC-CNPq), KSS (AT-CNPq), MMAQ (PIBIC-UA CNPq), AMA, ACSP, LCD, EOG, PSP and SGS (PIBIC-INPA-CNPq).

\section{LITERATURE CITED}

Anderson, J. E. et al. 1991. A blind comparison of simple bench-top bioassay and human tumour cell cytotoxicities as antitumor prescreens. Phytochemical Analysis, 2:107-111.

Badaway, A-Sam; Kappe, T. 1997. Potential antineoplastics. Synthesis and cytotoxicity of certain 4-chloro-3-(2-chloroethyl)2-methylquinolines and related derivatives. European Journal of Medicinal Chemistry, 32: 815-822.

Cepleanu, F. et al. 1994. Screening of tropical medicinal plants for molluscicidal, larvicidal, fungicidal and cytotoxic activities and brine shrimp toxicity. International Jour nal of Pharmacognosy, 32: 294-307. 
Table 2. Lethality of Plant Extracts in the Brine Shrimp Assay.

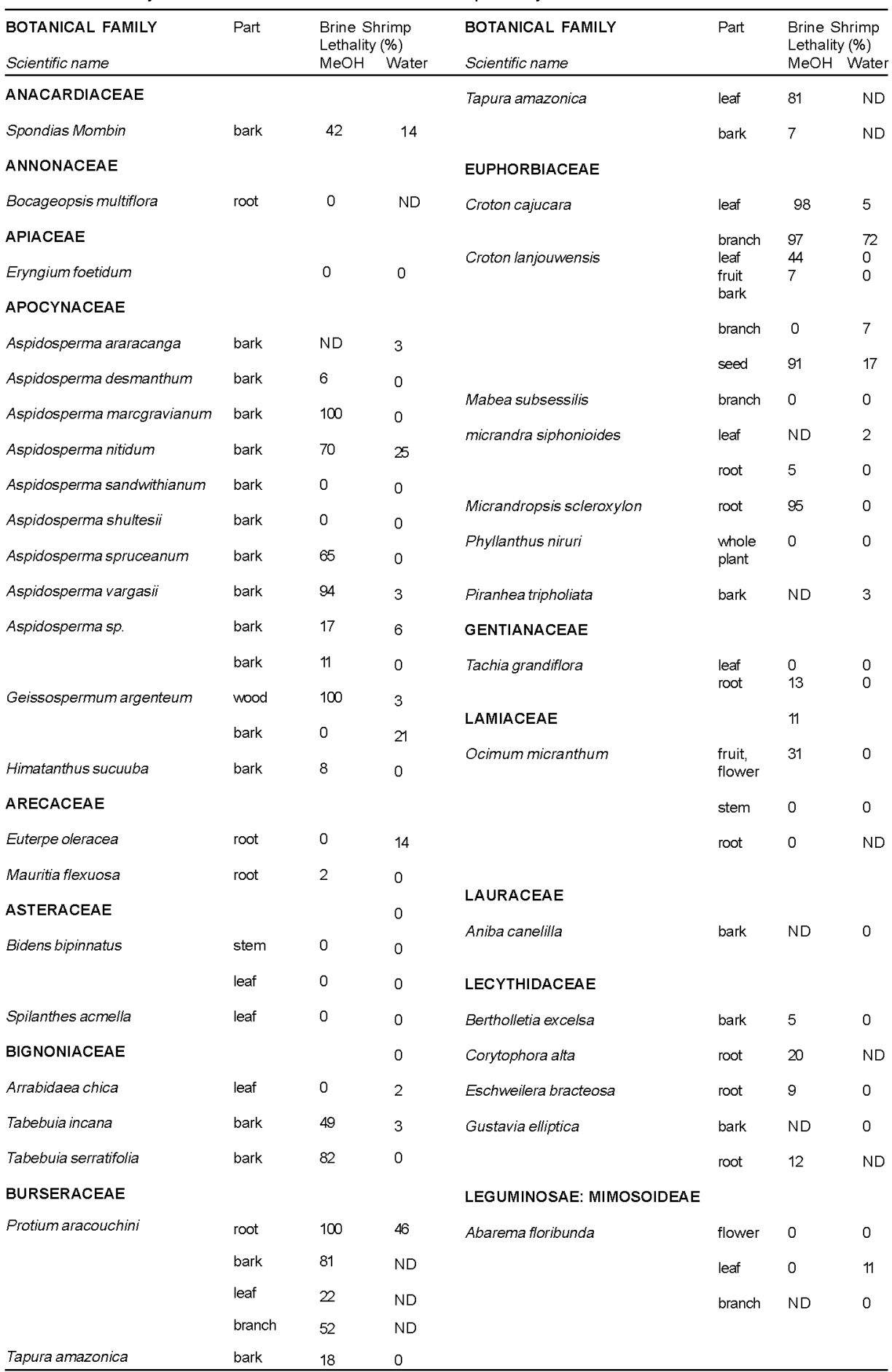


Table 2. Lethality of Plant Extracts in the Brine Shrimp Assay (continued).

\begin{tabular}{|c|c|c|c|c|c|c|c|}
\hline \multirow{2}{*}{$\begin{array}{l}\text { BOTANICAL FAMILY } \\
\text { Scientific name }\end{array}$} & \multirow[t]{2}{*}{ Part } & \multicolumn{2}{|c|}{$\begin{array}{l}\text { Brine Shrimp; } \\
\text { Lethality (\%) }\end{array}$} & \multirow{2}{*}{$\begin{array}{l}\text { BOTANICAL FAMILY } \\
\text { Scientific name }\end{array}$} & \multirow[t]{2}{*}{ Part } & \multicolumn{2}{|c|}{$\begin{array}{l}\text { Brine Shrimp } \\
\text { Lethality (\%) }\end{array}$} \\
\hline & & $\mathrm{MeOH}$ & Wets: & & & $\mathrm{MeOH}$ & Water \\
\hline ANACARDIACEAE & & & & Tapura amazonica & leaf & 81 & ND \\
\hline Spondias Mombin & bark & 42 & 14 & EUPHORBIACEAE & bark & 7 & ND \\
\hline Bocageopsis multiflora & root & 0 & ND & Croton cajucara & leaf & 98 & 5 \\
\hline $\begin{array}{l}\text { APIACEAE } \\
\text { Eryngium foetidum }\end{array}$ & & 0 & 3 & Croton lanjouwensis & $\begin{array}{l}\text { branch } \\
\text { leaf } \\
\text { fruit } \\
\text { barch }\end{array}$ & $\begin{array}{l}97 \\
44 \\
7\end{array}$ & $\begin{array}{l}72 \\
0 \\
0\end{array}$ \\
\hline APOCYNACEAE & & & & & branch & 0 & 7 \\
\hline Aspidosperma araracanga & bark & ND & 0 & & seed & 91 & 17 \\
\hline Aspidosperma desmanthum & bark & 6 & 0 & Mabea subsessilis & branch & 0 & 0 \\
\hline Aspidosperma marcgravianum & bark & 100 & 25 & micrandra siphonioides & leaf & ND & 2 \\
\hline Aspidosperma nitidum & bark & 70 & 0 & & root & 5 & 0 \\
\hline Aspidosperma sandwithianum & bark & 0 & 0 & Micrandropsis scleroxylon & root & 95 & 0 \\
\hline $\begin{array}{l}\text { Aspidosperma shultesii } \\
\text { Aspidosperma spruceanum }\end{array}$ & bark & 65 & 0 & Phyllanthus niruri & $\begin{array}{l}\text { whole } \\
\text { plant }\end{array}$ & 0 & 0 \\
\hline Aspidosperma vargasii & bark & 94 & 6 & $\begin{array}{l}\text { Piranhea tripholiata } \\
\text { GENTIANACEAE }\end{array}$ & bark & ND & 3 \\
\hline & bark & 11 & 0 & Tachia grandiffora & $\begin{array}{l}\text { leaf } \\
\text { root }\end{array}$ & $\begin{array}{l}0 \\
13\end{array}$ & $\begin{array}{l}0 \\
0\end{array}$ \\
\hline Geissospermum argenteum & wood & 100 & 3 & LAMIACEAE & & 11 & \\
\hline Himatanthus sucuuba & bark & 0 & $\begin{array}{l}21 \\
0\end{array}$ & Ocimum micranthum & $\begin{array}{l}\text { fruit } \\
\text { flower }\end{array}$ & 31 & 0 \\
\hline ARECACEAE & & & & & stem & 0 & 0 \\
\hline Euterpe oleracea & root & 0 & 14 & & root & 0 & ND \\
\hline Mauritia flexuosa & root & 2 & 0 & LAURACEAE & & & \\
\hline ASTERACEAE & & & & Aniba canelilia & bark & ND & 0 \\
\hline Bidens bipinnatus & $\begin{array}{l}\text { stem } \\
\text { leaf }\end{array}$ & 0 & $\begin{array}{l}0 \\
0\end{array}$ & LECYTHIDACEAE & & & \\
\hline Spilanthes acmella & leaf & 0 & 0 & Bertholletia excelsa & bark & 5 & 0 \\
\hline BIGNONIACEAE & & & & Corytophora alta & root & 20 & ND \\
\hline Arrabidaea chica & leaf & 0 & 2 & Eschweilera bracteosa & root & 9 & 0 \\
\hline Tabebuia incana & bark & 49 & 3 & Gustavia elliptica & bark & ND & 0 \\
\hline $\begin{array}{l}\text { Tabebuia serratifolia } \\
\text { BURSERACEAE }\end{array}$ & bark & 82 & 0 & LEGUMINOSAE: MIMOSOIDEAE & root & 12 & ND \\
\hline Protium aracouchini & root & 100 & 46 & Abarema floribunda & flower & 0 & 0 \\
\hline & bark & 81 & ND & & leaf & 0 & 11 \\
\hline & leaf & 22 & ND & & branch & ND & 0 \\
\hline Tapura amazonica & branch & 52 & ND & & & & \\
\hline
\end{tabular}


Table 2. Lethality of Plant Extracts in the Brine Shrimp Assay (continued).

\begin{tabular}{|c|c|c|c|c|c|c|c|}
\hline BOTANICAL FAMILY & Part & $\begin{array}{l}\text { Brine } \varsigma \\
\text { Lethalit }\end{array}$ & & BOTANICAL FAMILY & Part & $\begin{array}{l}\text { Brine S } \\
\text { Lethality }\end{array}$ & \\
\hline Scientific name & & $\mathrm{MeOH}$ & Water & Scientific name & & $\mathrm{MeOH}$ & Water \\
\hline LEGUMINOSAE: & & & & Piper amapense & leaf & 7 & ND \\
\hline & & & & & branch & 21 & 0 \\
\hline & leaf & 71 & 0 & & & & \\
\hline & hranch & 0 & & Piper capitarianum & leaf & 36 & ND \\
\hline & 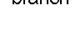 & & & & branch & 97 & ND \\
\hline & root & 0 & 42 & & mot & 97 & $N D$ \\
\hline Cassia siamea & flower & ND & 0 & & & & \\
\hline & ext & 2 & ? & Piper cyrtopodum & branch & 4 & ND \\
\hline & & & & Piper erectipilum & leaf & 0 & ND \\
\hline & branch & 0 & 0 & Piperhostmannianum & leaf & 95 & 6 \\
\hline Cassia spruceana & bark & 0 & 17 & & branch & 72 & 0 \\
\hline & root & 0 & 5 & Piper tuberculatum & leaf & 95 & 3 \\
\hline Senna occidentalis & leaf & 9 & 70 & & fruit & 100 & 58 \\
\hline Senna reticulata & flower & 16 & 0 & & branch & 100 & 68 \\
\hline & leaf & 0 & 0 & & & & \\
\hline & stem & 65 & 3 & Pothomorphe peltata & leat & 14 & 0 \\
\hline & & & & & fruit & 75 & 0 \\
\hline & pod & 0 & 0 & & branch & 34 & 0 \\
\hline & branch & 0 & 0 & & & & \\
\hline & root & 0 & 0 & & 1000 & & 0 \\
\hline $\begin{array}{l}\text { LEGUMINOSAE: } \\
\text { PAPILIONOIDEAE }\end{array}$ & & & & POACEAE & & & \\
\hline Bowdichia nitida & & & & Cymbopogon citratus & leaf & 50 & 0 \\
\hline Swartizia pronacencis & root & ND & 0 & RHAMNACEAE & & & \\
\hline Sover & root & 2 & 0 & Ampelozizyphus amazonicus & & 31 & 11 \\
\hline MENISPERMACEAE & & & & & & & \\
\hline Abuta grandifolia & & 2 & 0 & RHIZOPHORACEAE & & & ND \\
\hline & vine & 22 & 11 & Cassiopourea quianensis & $m$ & 25 & ND \\
\hline & $\begin{array}{l}\text { Whole } \\
\text { plant }\end{array}$ & 27 & 0 & RUBIACEAE & & & 0 \\
\hline OLACACEAE & & & & & & & \\
\hline & & & & Ladenbergia undata & bark & 22 & 8 \\
\hline Wimquarla guantiensis & bark & 96 & 3 & Palicourea carymbifera & leaf & 0 & ND \\
\hline & branch & & & & stem & ND & 0 \\
\hline Agonandra brasiliensis & & 5 & & 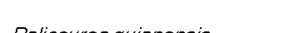 & 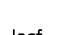 & 0 & 0 \\
\hline Petiveria alliacea & root & 5 & ND & Palicourea guianensis & leaf & 0 & 0 \\
\hline & leaf & 0 & 0 & Palicourea virens & leaf & 0 & 0 \\
\hline & root, & 0 & 0 & & stem & ND & 0 \\
\hline & stem & ND & 0 & RUTACEAE & & & \\
\hline PIPERACEAE & & & & Citrus limetta & mot & 100 & 0 \\
\hline Piper aduncum & & & & & & & \\
\hline & stem & 7 & 0 & SAPOTACEAE & & & \\
\hline & leaf & 100 & 0 & Micropholis venulosa & root & 8 & 27 \\
\hline & branch & 0 & 0 & & bark & ND & 0 \\
\hline & root & 100 & 0 & & root & 8 & ND \\
\hline
\end{tabular}


Table 2. Lethality of Plant Extracts in the Brine Shrimp Assay (Continued).

\begin{tabular}{|c|c|c|c|c|c|c|c|}
\hline \multirow{2}{*}{$\begin{array}{l}\text { BOTANICAL FAMILY } \\
\text { Scientific name } \\
\text { Pouteira caimito }\end{array}$} & \multirow{2}{*}{$\begin{array}{l}\text { Part } \\
\text { bark }\end{array}$} & \multicolumn{2}{|c|}{$\begin{array}{l}\text { Brine Shrimp } \\
\text { Lethality (\%) } \\
\text { MeOHWater }\end{array}$} & \multirow{2}{*}{$\begin{array}{l}\text { BOTANICAL FAMILY } \\
\text { Scientific name } \\
\text { Simaba cedron }\end{array}$} & \multirow{2}{*}{$\begin{array}{l}\text { Part } \\
\text { stem }\end{array}$} & \multicolumn{2}{|c|}{$\begin{array}{l}\text { Brine Shrimp } \\
\text { Lethality (\%) } \\
\text { MeOH Water }\end{array}$} \\
\hline & & 5 & 5 & & & 42 & 58 \\
\hline Pouteria guianensis & root & 13 & 5 & & leaf & 0 & 0 \\
\hline \multicolumn{8}{|l|}{ SIMAROUBACEAE } \\
\hline \multirow[t]{8}{*}{ Picramnia spruceana } & leaf & 6 & 7 & & & & \\
\hline & minor & 0 & 19 & Physalis angulada & root & 0 & 0 \\
\hline & branch & & & VIOLACEAE & & & \\
\hline & $\begin{array}{l}\text { trunk } \\
\text { wood }\end{array}$ & 0 & 0 & Rinorea guanensis & bark & ND & 0 \\
\hline & petiole & 2 & 24 & & & & \\
\hline & seed & 0 & 0 & & leaf & ND & 3 \\
\hline & & & & & root & 3 & ND \\
\hline & & & & Rinorea racemosa & root & 0 & 0 \\
\hline
\end{tabular}

$N D=$ Not determined. Lethalities should be considered accurate to within $\pm 3 \%$.

Duke, J. A.; Vasquez, R. 1994. Amazonian

- Ethnobotanical Dictionary. Florida, United States: CRC Press, 215 p.

Fatope, M.O. et al. 1993. Screening of higher plant reputed as pesticides using the brine shrimp lethality assay. International Journal of Pharmacognosy, 31: 250-254.

Grenand, P.; Moretti, C.; Jacquemin, H. 1987. Pharmacopées Traditionnelles en Guyane: Créole, Palikur, Wayãpi. Paris: ORSTOM, $569 \mathrm{p}$.

Labbe, C.; Castillo, M.; Connoly, J.D. 1993. Mono and sesquiterpenoids from Satureja gilliesii. Phytochemistry, 34: 441-444.

Le Cointe, P. 1947. Árvores e Plantas úteis (Indigenas e Aclimadas). São Paulo: Compania Editora Nacional, 506 p.

Lorenzi, H. 1998. Árvores Brasileiras: Manual de Identificação e Cultivo de Plantas Arboreas Nativas do Brasil. Nova Odessa, São Paulo: Plantarum, 352 p.
McLaughlin, J.L.; Chang, C.-J.; Smith, D.L. 1991. "Bench-top" bioassays for the discovery of bioactive natural products: an update. In: Rhaman, A.U. (ed.). Studies in Natural Product Chemistry, v. 8, Elsevier, Amsterdam.

Meyer, B.N. et al. 1982. Brine shrimp: a convenient general bioassay for active plant constituents. Planta Medica, 45: 31-34.

Milliken, W. 1997. Plants for Malaria, Plants for Ferver. Medicinal species in Latin America - a bibliographic survey. Dickeson, S. (ed.). The Royal Botanic Garden, Kew (U.K.), 116 p.

Mongelli, E. et al. 1996. Screening of Argentine medicinal plants using the bine shrimp microwell ctotoxicity assay. International Journal of Pharmacognosy, 34: 249-254.

Mors, W. B.; Rizzini, C. T.; Perreira, N. A. 2000. Medicinal Plants of Brazil. DeFfilipps, R. A (ed.). Reference Publication, Inc., Algonac (U.S.A.), 501 p. 
Oberlies, N.H. et al. 1998. Cytotoxic and insecti cidal constituents of the unripe fruit of Persea americana. Journal of Natural Products, 61 : 781-785.

Ohashi, M.; Oki, T. 1996. Ellipticine and related anticancer agents. Expert Opinion on Therapeutic Patents, 6: 1285-1294.

Parmar, V.S. et al. 1997. Phytochemistry of the genus Piper. Phytochemistry, 46: 597-673.

Parra, A.L. et al. 2001. Comparative study of the assay of Artemia salina L. and the estimate of the medium lethal dose ( $\mathrm{LD}_{50}$ value) in mice, to determine oral acute toxicity of plant extracts. Phytomedicine, 8: 395-400.

Pérez, H.; Díaz, F.; Medina, J. D. 1997. Chemical investigation and in vitro antimalarial activity of Tabebuia ochracea ssp. neochrysantha. International Journal of Pharmacognosy, 35 : 227-231.

Pio Corrêa, M. 1978. Dicionário das Plantas Úteis do Brasil e das Exóticas Cultivadas. Rio de Janeiro: Ministério da Agricultura-Instituto Brasileiro de Desenvolvimento Florestal, v. I-VI.
Revilla, J. 2002. Apontamentos para a Cosmética Amazônica. Manaus: SEBRAE-AM / INPA, $532 \mathrm{p}$.

Ribeiro, J. E. L. da S. et al. 1999. Flora da Reserva Ducke: Guia de Identificação das Plantas Vasculares de uma Floresta de Terra-firme na Amazônia Central. Manaus: INPA / DFID, 800 p.

Schultes, R.E.; Raffauf, R.F. 1990. The Healing Forest: Medicinal and Toxic Plants of the Northwest Amazonia. Oregon, United States: Dioscorides Press, 484 p.

Silva, M. F.; Lisbôa, P. L. B.; Lisbôa, R.C.L. 1977. Nomes vulgares de plantas Amazônicas. Manaus: INPA, 222 p.

Siqueira, J. M. et al. 2001. Estudo fitoquímico das cascas do caule de Duguetia glabriuscula - Annonaceae, biomonitorado pelo ensaio de toxicidade frente a Artemia salina Leach. Quimica Nova, 24:185-187.

Submetido à publicação: 22/04/2002

Aceito: 29/10/2002 